\title{
GETA sandals: a footstep location tracking system
}

\author{
Shun-yuan Yeh $\cdot$ Keng-hao Chang • \\ Chon-in Wu - Hao-hua Chu $\cdot$ Jane Yung-jen Hsu
}

Received: 11 August 2005/ Accepted: 6 December 2005/Published online: 1 February 2007

(C) Springer-Verlag London Limited 2007

\begin{abstract}
This paper presents the design, implementation, and evaluation of a footstep based indoor location system. The traditional Japanese GETA sandals are equipped with force, ultrasonic, orientation, RFID sensors and an accelerometer to produce a wearable location tracking system that demand little infrastructure in the deployed environment. In its basic form, a user simply puts on GETA sandals to enable tracking of his/her locations relative to a starting point (e.g., a building entrance), making it easy for deployment everywhere. The footstep location system is based on dead-reckoning, which works by measuring and tracking displacement vectors along a trail of footsteps. Each displacement vector is formed by drawing a line between each pair of footsteps, and the position of a user can be calculated by summing up the current and all previous displacement vectors. Unlike most existing
\end{abstract}

\footnotetext{
S.-y. Yeh · C.-i. Wu · H.-h. Chu $(\bowtie)$ · J. Y.-j. Hsu

Department of Computer Science and Information Engineering, Graduate Institute of Networking and Multimedia, National Taiwan University, \#1 Roosevelt Road, Section 4, Taipei 106, Taiwan e-mail: hchu@csie.ntu.edu.tw

S.-y. Yeh

e-mail: r93124@csie.ntu.edu.tw

C.-i. $\mathrm{Wu}$

e-mail: r92079@csie.ntu.edu.tw

J. Y.-j. Hsu

e-mail: yjhsu@csie.ntu.edu.tw

K.-h. Chang

Department of Electrical Engineering

and Computer Sciences, University of California,

Berkeley, USA

e-mail: kenghao@cs.berkeley.edu
}

indoor location systems, the footstep based method does not suffer from problems with obstacles, multipath effects, signal noises, signal interferences, and dead spots. There are two technical challenges in the proposed design: (1) location error accumulates over distance traveled, and (2) displacement measurements are sporadic during stair climbing. The first problem is addressed by a light RFID infrastructure, while the second problem is remedied by incorporating an accelerometer into the system. Experiments on GETA prototype are conducted to evaluate the positional accuracy of our system.

\section{Introduction}

Physical locations of people and objects are the most widely used context information in context-aware applications. To enable location-aware applications in indoor environments, many indoor location systems such as Active Badge [1], Active Bat [2], Cricket [3], Smart Floor [4], RADAR [5], and Ekahau [6] were proposed in the past decade. However, there is no widespread adoption of such systems in everyday environments. We believe that the main obstacle is the level of system infrastructural support required in the deployment including hardware, installation, calibration, maintenance, etc. Significantly reducing the needed system infrastructure serves as our primary motivation to design and prototype a new footstep location system. The traditional Japanese GETA (pronounced "gue-ta") sandals are outfitted to compute a user's physical location with multiple sensors installed onboard and without other infrastructure. All you need are sandals. 
This basic idea can be understood by looking at a person leaving a trail of footprints on a beach. The system continuously measures a displacement vector formed between two footsteps advancing temporally and spatially. To track a person's physical location relative to a starting point, the system simply sums up all previous footstep displacement vectors leading to his/her current location. This idea is similar to the dead-reckoning navigation, which dates back to the medieval time, when sailors or navigators located themselves by measuring the course and distance sailed from a starting point. In our system, we adapted deadreckoning to track human footsteps from a starting point, such as the entrance of an indoor facility. We believe that having a wearable location tracker is an important advantage over infrastructure-based indoor location systems. Users simply wear GETA location system, and walk anywhere they want to go.

In addition to the benefit of low infrastructure cost, the footstep location system does not have problems commonly found in other indoor location systems. Existing wireless solutions based on radio, ultrasound, or infrared need a sensing range large enough to cover the distance between fixed location sensors in the environment and a mobile location sensor worn by the user. Such systems often experience poor positional accuracy when encountering obstacles between transmitters and receivers, multi-path effects, signal interferences, and dead spots.

In contrast, these problems are less likely to appear in our location system. The ultrasonic-RF transmitters and receivers in the proposed footstep method are designed to cover only the distance between two sandals, up to a maximum length of a walking step $(<1.5 \mathrm{~m})$. Given such a small sensing range, sensing problems are relatively small. The range also brings two additional advantages: (1) the power consumption of the location sensors can be significantly reduced, and (2) the ultrasonic location sensors have high accuracy. However, the footstep location tracking system also comes with two main shortcomings: (1) an error accumulation problem and (2) a stair climbing limitation. Below we briefly describe these two shortcomings and our remedies.

Error accumulation problem Each time when the footstep-based method takes measurement to calculate a displacement vector, a small amount of error is introduced. Consider a user who walks $n$ steps away from a starting point, his/her current location is calculated as a sum of these $n$ displacement vectors. This means that the current positional error is the sum of all errors from these $n$ previous displacement vectors as a percentage of the total distance traveled. Our previous work [7] addressed this error accumulation problem by utilizing a small RFID reader placed under a GETA sandal to read a small number of passive RFID tags in the environment with known coordinates. Encountering a RFID tag has the same effect as resetting the accumulated error to zero. Although these locationaware RFID tags are considered as a system infrastructure, they constitute a very light infrastructure because RFID tags (1) are relatively inexpensive $(<\$ 1$ each), (2) are easy to install, and (3) are only needed in a very small numbers. Based on our experimental results described in Sect. 5, to limit the average error to $1 \mathrm{~m}$, we need to install enough RFID tags in the environment such that a user is likely to walk over a RFID tag approximately every 27 steps or $8 \mathrm{~m}$.

Stair climbing limitation This occurs where stairs become obstacles blocking ultrasonic pulse transmission between two sandals. Under this situation, the ultrasonic sensor cannot measure a displacement vector between two advancing footprints. To address this limitation, our localization system incorporates an accelerometer-based method to calculate step vectors from acceleration data collected on one of GETA sandals. Although the accelerometer-based method has lower accuracy than the ultrasound-based method, it is a good complement and backup method to improve overall robustness of the system.

The remainder of this paper is organized as follows. Section 2 discusses related work. Section 3 describes the design of our footstep method. Section 4 explains our prototype implementation of GETA sandals, and Sect. 5 discusses experimental results. Finally, Sect. 6 draws our summary and suggests future work.

\section{Related work}

We organize the related work into the following two categories: infrastructure and non-infrastructure supported indoor location systems. Infrastructure supported systems include Active Badge [1], Active Bat [2], Cricket [3], and Ubisense [8]. These systems are based on signal time-of-flight measurement to estimate distances to certain fixed positions in the environment and to apply the triangulation method to compute spatial coordinates. In other words, these systems generally require installation of a number of infrared, ultrasonic, or ultra-wideband transmitters (or receivers) at fixed locations (e.g., ceilings or high walls). For example, Active Bat system [2] requires installing ultrasonic receivers in the environment and an ultrasonic transmitter on a mobile bat unit worn by each user. Ubisense [8] is a commercial product based on 
ultra-wideband (UWB) technology. Proponents of UWB technology claim that it has many advantages, such as resistance to multi-path fading, penetration of walls and other solid objects, and interoperability with other radio based systems. In order to attain high location accuracy and good spatial coverage, the system infrastructure requires a number of transmitters (or receivers) installed. Ordinary people maybe unable to afford, operate, and maintain the infrastructure in their everyday environments.

Many infrastructure supported systems have been developed using Wi-Fi RSSI values to estimate locations. These systems can be categorized into two broad approaches. The first approach is based on the deterministic method [5, 6, 9, 10]. Systems following this approach apply deterministic inference, such as triangulation and $k$-nearest-neighbors (KNN) search, to estimate the target device's location. For example, the RADAR system [5] applies KNN to obtain the $k$ nearest neighbors and estimates the location of the target device by averaging the locations of these $k$ nearest neighbors. Ekahau [6] is commercial product software based on similar ideas.

The second approach is based on the probabilistic method [10-13]. Seshadri et al. [10] applied Bayesian inference, which uses multiple probabilistic models and histograms to enhance the performance of location systems. This method calculates conditional probabilities over locations based on RSSI values. Some probabilistic methods also add a motion model to describe the continuity in human's movements such that they can lower the oscillatory location estimations in Wi-Fi based localization systems. These $\mathrm{Wi}$-Fi based systems require an existing Wi-Fi network. For example, the Ekahau location system recommends a Wi-Fi client to be able to receive signals from 3 to $4 \mathrm{Wi}-\mathrm{Fi}$ access points in order to attain the specified positional accuracy of $3 \mathrm{~m}$. This high density of access points is unlikely in our everyday home and small office environments. In addition, most Wi-Fi based location systems require users' calibration efforts to construct a radio map by taking measurements of signal strength at various points forming another barrier for users.

Furthermore, the instability caused by dynamic environmental factors can also reduce the positional accuracy and stability in the Wi-Fi location systems [15]. Smart floor [4] can track the location of a user by using pressure or presence sensors underneath the floor tiles to detect the user's gait. It also attempts to determine user identity using the biometric signature of a person's footsteps. This infrastructure is expensive because it requires custom-made floor tiles and flooring re-construction.
The second category of related work is non-infrastructure supported indoor positioning system. Most of the systems are based on incremental motion and dead reckoning. This is well suited for instrumented vehicles such as aircraft, ships, and automobiles [16]. On a smaller scale, dead reckoning is also used for robot navigation [17]. The use of motion sensors for virtual and augmented reality head trackers is commonplace, demonstrating that these sensors can provide accurate position prediction in small areas [18, 19].

Lee et al. [20] propose a method to estimate a user's current location by recognizing a sequence of incremental motions (e.g., 2 steps north followed by 40 steps east, etc.) from wearable sensors such as accelerometers, digital compass, etc. Lee's method differs from our footstep tracking system in that it can only recognize a few selected locations (e.g., bathroom, toilet, etc.) rather than track location coordinates. Point research [21] provides a vehicle self-tracking system that generates high location accuracy by combining the dead-reckoning method (wheel motions) and GPS. Our method differs from the solution from Point research solution because ours is based on footstep tracking in normal human walking motion rather than on mechanical wheel movements.

Several RFID-based localization systems are proposed. NaviGETA [22] embeds a RFID reader in a sandal to sense location-aware RFID tags in the environment. Willis et al. [23] propose a location determination system for the blind by embedding a RFID reader in a walking cane to sense a passive RFID tag grid. Amemiya et al. [24] build an active RFID tag grid to position users.

\section{Design}

The design of our footstep location system is based on observations of real human walking motion. Human walking motion can be modeled by stance-phase kinematics shown in Fig. 1. A forwarding walking motion consists of a sequence of three stances-heelstrike, mid-stance, and toe-off. In the heel-strike stance, the body weight pushes down from the upper body to the lower body, strike the heel (right), and resulting in both feet touch on the ground. This generates a footstep. In the mid-stance, the body raises one (left) foot moving it forward and above the ground. In the toe-off stance, the body weight again pushes down on the forwarded (left) foot with another heel strike and lifts the back (right) foot in a toe-off stance, again resulting in both feet in contact with the ground. This generates another footstep. 
Fig. 1 Three stances in a normal human walking motion

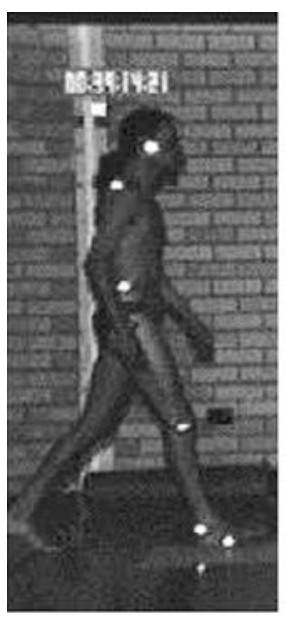

Heel-strike

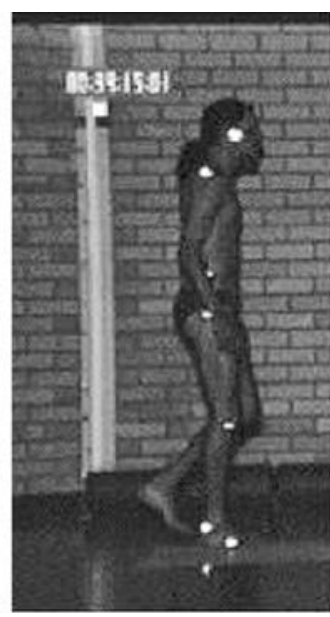

$>$ Mid-stance

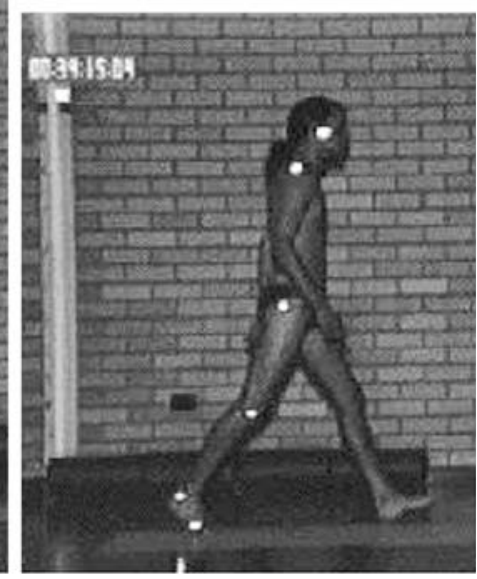

Toe-off
The basic idea behind our footstep location tracking system is to (1) detect heel-strike and toe-off stances, when the both sandals are on the ground then (2) take measurement of the step displacement vector.

We incorporate two different distance measurement methods to estimate step displacement vectors at the moment of heel-strike and toe-off stances. The first method is an ultrasound-based method shown in Fig. 2a. It measures step vectors from ultrasonic sensors installed in both left and right sandals. Specifically, each step vector is measured from the left sandal to the right sandal. The second method is an accelerometerbased method shown in Fig. 2b. It measures step vectors from an accelerometer sensor installed underneath (a)

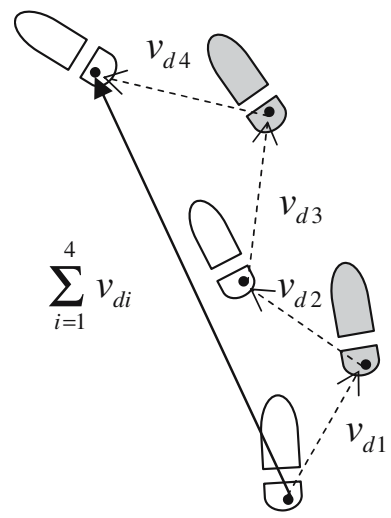

Starting point $\left(x_{\text {start }}, y_{\text {start }}\right)$ (b)

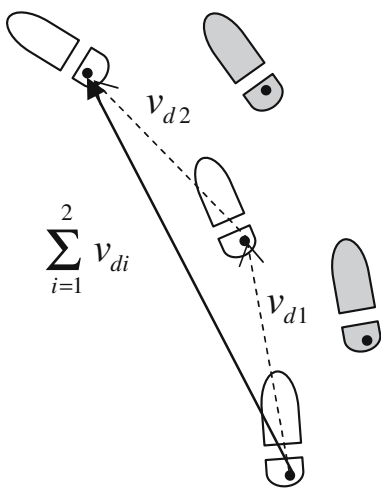

Starting point $\left(x_{\text {start }}, y_{\text {start }}\right)$
Fig. 2 The two methods to measure the user's foot step vector. The user has walked four footsteps 1-4. a It shows how ultrasound-based method measures vectors and how the vectors results in final user location. b It shows the case of accelerometer-based method a left sandal. Specifically, each step vector is measured from a left sandal's previous position to its current position. Note that our footstep system runs both distance measurement methods concurrently and independently, and so that they complement each other in different environmental settings.

Relative to a starting point ( $\left.x_{\text {start }}, y_{\text {start }}\right)$ shown in Fig. 2 (e.g., an entrance of an indoor facility), our footstep system tracks the current position of a user, who has walked $n$ number of steps away from the starting point, by summing up the starting point and all displacement vectors $\Sigma v_{d i}$, for $i=1, \ldots, n$, where $n$ is number of vectors measured. These two distance measurement methods (ultrasound-based and accelerometer-based) are described in details below.

\subsection{Ultrasound-based method}

Our ultrasound-based method is designed to take advantage of normal human walking characteristics. Under normal walking motion, a person only moves one foot at a time while the other foot stays fixed. Therefore, we can use the fixed foot as a reference point to measure a new position (step vector) of a forwarding foot. This is quite different from ordinary self trackers which treat a user's entire body as a whole to estimate position by kinematical state [21].

To realize this design, two force sensors are placed at the bottom of both sandals to detect heel-strike and toe-off stances. After heel-strike and toe-off stances are detected, we measure a step vector $v_{d}$ for the current footstep. To measure this step vector, two ultrasonic-RF-combo receivers are placed on the left sandal and one ultrasonic-RF-combo transmitter is placed on the right sandal shown in Fig. 3. Transmitters and 
receiver are positioned on the inner sides of the sandals and aligned face-to-face toward each other to enable range estimation. The prototype of GETA sandals is shown in Fig. 7.

Step vectors are measured in the following two steps. First, by the time-of-flight method we can measure two distances from one ultrasonic transmitter to two receivers shown in Fig. 3. Then by applying triangulation, we can obtain the $(x, y)$ coordinate of the transmitter located on the right sandal. Note that the obtained coordinate of the transmitter is only relative to the local coordinate system of the left sandal, where the origin of this local coordinate system is at the position of the receiver ${ }_{2}$ and the $X$-axis forms a straight line from the receiver ${ }_{2}$ to the receiver $_{1}$. Since the movement of left sandal rotates the local coordinate system, re-orientating the step vector from its local coordinate system back to a global coordinate system is necessary, where the global coordinate system is set to be the coordinate system at the starting point. To perform this re-orientation, an orientation sensor is used to sense the orientation angle $\theta$ of the local coordinate system relative to the global coordinate system shown in Fig. 3 by the gray arrows. Finally, the step vector $v_{d}$ (the black solid arrow in Fig. 3) can be computed using the following equation:

$v_{d}=\left[\begin{array}{cc}\cos \theta & \sin \theta \\ -\sin \theta & \cos \theta\end{array}\right]\left[\begin{array}{l}x \\ y\end{array}\right]$.

This method, however, has two limitations. The first limitation occurs when obstacles lie between the two sandals and block ultrasonic pulses from the transmitter to receivers. For example, during stair climbing, stairs become obstacles. The second limitation occurs when both feet move simultaneously. For example, during a jumping motion, both feet are lifted off the ground and then touch the ground at approximately the same time. In this case, there is no fixed point as a reference from

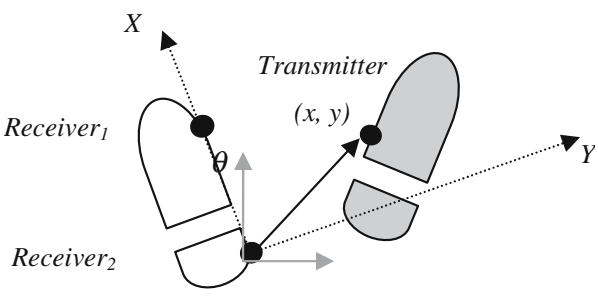

Fig. 3 Figure shows the locations of ultrasonic-RF receivers and transmitter on the sandals. The coordinate of the transmitter on the right sandal is relative to the local coordinate system on the left sandal which the position of a moving foot can be measured. To address these two limitations, the footstep method incorporates a complementary distance measurement method based on an accelerometer. The accelerometerbased method is described below.

\subsection{Accelerometer-based method}

The basic idea of this method is to make the left sandal into a self tracker. When a user moves his/her left foot, a displacement vector $v_{d}$ is measured on his/her left sandal as shown in Fig. 2b. To perform such measurements, the following hardware components are installed on the left sandal: (1) a dual-axis accelerometer, (2) a 3D orientation sensor, and (3) a force sensor.

Figure 4 shows how the accelerometer-based method works. There are three steps: (1) the force sensor is used to detect when the left sandal is lifted off the ground, indicating the start of a walking motion; (2) once the left sandal is off the ground, synchronized streams of acceleration and orientation data are collected at the rate of 100 samples per second $(100 \mathrm{~Hz})$ until the force sensor detects that the left sandal is on the ground again, i.e., a user has completed a left step; and (3) a step displacement vector is calculated from the collected data by performing acceleration compensation and double integral algorithms, which are explained in detail below.

\subsubsection{Acceleration compensation}

The goal of acceleration compensation is to filter out the effect of gravitation from acceleration readings, leaving only clean acceleration produced by moving motion. This clean acceleration is then used as input to double integral algorithm to calculate step displacement vectors.

During a normal human walking motion, sandal unavoidably becomes tilted from the horizontal plane from time to time. When a sandal is tilted, its accelerometer, fixed flatly underneath the sandal, also becomes tilted. This causes raw accelerometer reading to be affected by a combination of (1) horizontal acceleration produced by a sandal's walking motion and (2) gravitational acceleration introduced by a sandal's tilting from the horizontal plane, e.g., angles $\theta_{\text {Pitch }}$ and $\theta_{\text {Roll }}$, as illustrated in Fig. 5. Since gravitational acceleration does not contribute to any distance forward, it is unwanted noise. To filter out gravitational noise, a $3 \mathrm{D}$ orientation sensor is used to sense the tilting angles $\theta_{\text {Pitch }}$ and $\theta_{\text {Roll }}$. The following equations are used to obtain the pure horizontal acceleration $a_{x}^{\prime}$ and $a_{y}^{\prime}$ from the raw acceleration data $a_{x}$ and $a_{y}$ : 
Fig. 4 Procedure of calculating step displacement vector in the accelerometerbased method

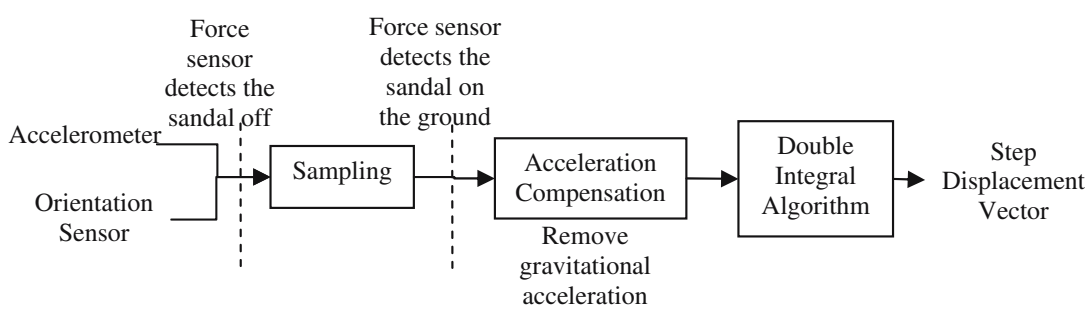

$a_{x}^{\prime}=\left(a_{x}+g \times \sin \left(\theta_{\text {Pitch }}\right)\right) \cos \left(\theta_{\text {Pitch }}\right)$,

$a_{y}^{\prime}=\left(a y-g \times \sin \left(\theta_{\text {Roll }}\right)\right) \cos \left(\theta_{\text {Roll }}\right)$.

The variables $a_{x}$ and $a_{y}$ are raw acceleration readings on the $x$ and $y$ axes from the accelerometer; $a_{x}^{\prime}$ and $a_{y}^{\prime}$ are the filtered acceleration readings, free from gravitational acceleration, on the $x$ and $y$ axes; and $g$ is the gravitational acceleration. The term $(g \times \sin (\theta))$ represents the portion of gravitational acceleration produced from a pitch or a roll. The term $\cos (\theta)$ is used to project the amount of gravitational acceleration onto the horizontal plane.

\subsubsection{Double integral algorithm}

Since accelerometer readings are discrete-time signals, they can be modeled as discrete-time points on a nonlinear curve corresponding to the movement path of a forward moving footstep shown in Fig. 6. Each discrete-time point contains the following information $\left(a_{x}^{\prime}, a_{y}^{\prime}, \theta_{\text {Yaw }}\right)$, where $\left(a_{x}^{\prime}, a_{y}^{\prime}\right)$ are the filtered, clean acceleration of a sandal in the $x$ and $y$ directions, and $\theta_{\text {Yaw }}$ is the direction of the acceleration on the

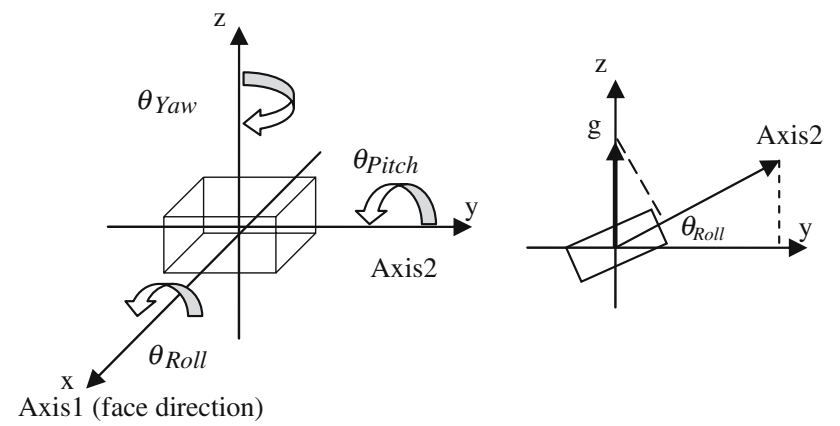

Fig. 5 Acceleration compensation. A dual-axes accelerometer is placed flatly on axis-1/axis-2, parallel to the horizontal plane $(x-y$ plane). It measures accelerations in both directions. When the sandal is off the ground, sandal tilting causes the accelerometer to rotate in a roll and a pitch direction, creating an angle $\theta_{\text {Pitch }}$ on the axis- 1 and an angle $\theta_{\text {Roll }}$ on the axis- 2 to the horizontal plane. For example, sandal tilting creates an angle $\theta_{\text {Roll }}$ between the axis- 2 and the $y$-axis shown on the right drawing. Therefore, acceleration compensation needs to remove the gravitational acceleration $g \times \sin \left(\theta_{\text {Roll }}\right)$ from the raw acceleration reading horizontal plane obtained from an orientation sensor. A full step displacement vector (shown as the black solid arrow in Fig. 6) can be calculated as the sum of these multiple piece-wise displacement vectors (shown as black dashed arrows in Fig. 6), which are in turn computed by applying double-integral using the trapezoidal rule on two successive acceleration data points and rotated to the global coordinate system by $\theta_{\text {Yaw }}$.

The advantage over the ultrasound-based method is that the accelerometer-based method is not limited by between-feet obstacle and it works on stairs. In other words, the accelerometer-based method is more robust. However, the disadvantage of the accelerometerbased method is that it has lower positioning accuracy, because direct distance measurement by ultrasound is more accurate than discrete-time double integral by acceleration.

\subsection{Combining ultrasound- and accelerometer- based methods}

Our footstep location tracking system uses these two distance measurement methods to obtain better accuracy and robustness. In this hybrid system both distance

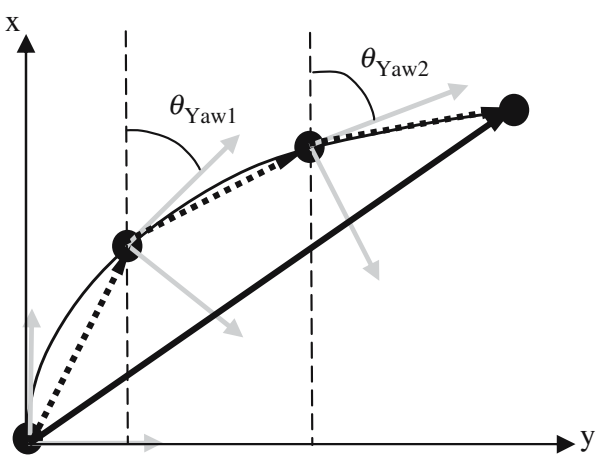

Fig. 6 Double integral algorithm. The black nonlinear curve is a trace of a turning right motion. The black points are the sampled acceleration points. The gray arrows represent the directions of the sampled acceleration. The black solid arrow is the full step displacement vector. The piece-wise displacement vectors between each point are the black dashed arrows. The step displacement vector is the sum of all piece-wise displacement vectors 
Fig. 7 Prototype

implementation of GETA sandals
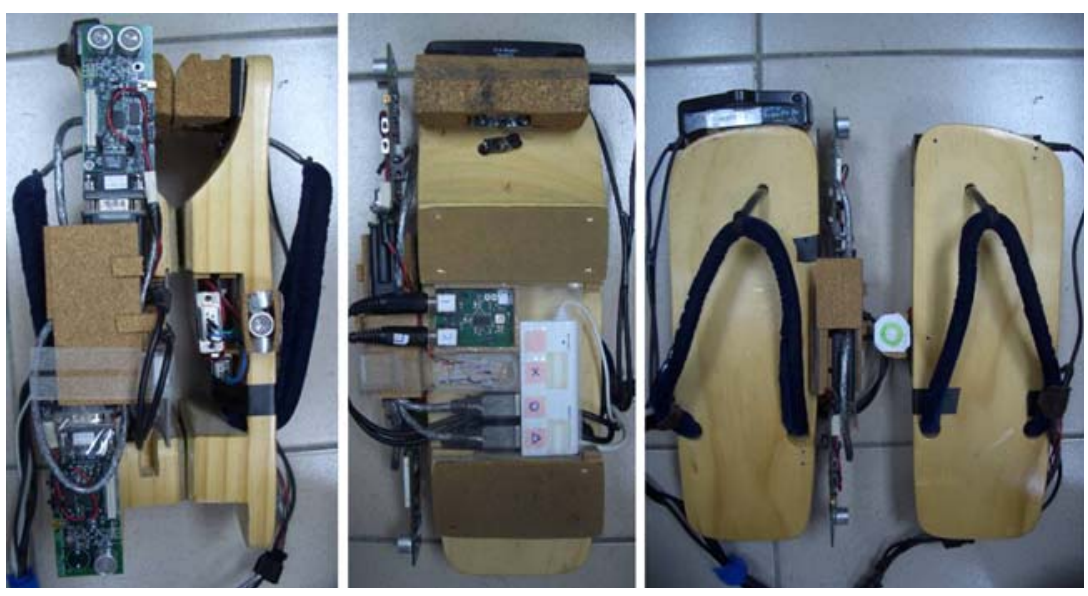

measurement methods are executed concurrently and independently. If the system cannot successfully transmit and receive ultrasonic pulse for distance measurement (e.g., caused by an obstacle between sandals), the less accurate step vector calculated from the accelerometer-based method is used; otherwise, the more accurate step vector calculated from the ultrasound-based method is used. In the future, both measurements may actually be combined into one vector to further increase accuracy.

\subsection{Passive RFID-based calibration}

Our footstep method, like other dead-reckoning methods, has an error accumulation problem. To address this error accumulation problem, a passive RFID-based calibration method is proposed. It consists of location-aware passive RFID tags, installed in the deployed environment, and a RFID reader, installed in a sandal, to reset any accumulated error whenever a user steps on a RFID tag with a known location coordinate. These location-aware passive RFID tags form a passive RFID grid to bound accumulated error. A higher density RFID grid implies a higher probability for a user to step on a passive RFID tag and to reset the accumulated error, but it also means larger numbers of RFID tags and higher cost. For a given location-aware application with certain accuracy and precision requirements, an ideal density of the RFID grid can be chosen to meet the application's requirements. For example, if an application demands $x$ meters (e.g., $3 \mathrm{~m}$ ) of positional accuracy and the sandal's positional error is measured as $y$ percent $(10 \%)$ of the distance traveled, the RFID grid density can be set so that a user will hit a tag in approximately every $100 x / y$ meters $(3 / 0.1=30 \mathrm{~m})$.

Passive RFID-based calibration utilizes a server to maintain the table mappings between RFID tag IDs and corresponding location coordinates. When a user enters the target environment, his/her GETA sandal downloads this mapping table to its local storage. After a GETA sandal steps on top of a RFID tag, it looks up this mapping table to find the location coordinate of this RFID tag. Then, the current location of a user is set to the location coordinate of this RFID tag rather than by the footstep tracking method.

\section{Prototype implementation}

We implemented the footstep location tracking method on a pair of GETA sandals as shown in Fig. 7. Five types of sensors are used in this prototype: (1) two force sensors, (2) an ultrasonic-RF-combo transmitter and two ultrasonic-RF-combo receivers, (3) a dual-axis accelerometer, (4) a 3D orientation sensor, and (5) a RFID reader. All sensor components are hardwire connected to a small Palm-top PC.

Force sensors are from Phidgets [25], and they are fixed underneath the toe of sandals. The maximum load on these force sensors is $3 \mathrm{~kg}$. To detect heelstrike and toe-off stances (i.e., both sandals are on the ground), a pressure threshold value is used to determine the change in the state of a sandal from being on the ground or in the air. This pressure threshold, the point at which the foot leaves the ground, is chosen as $30 \mathrm{~g}$ based on experimental trials-and-errors with our sandals. A $0 \mathrm{~g}$ point is not chosen because of measurement noise. Figure 8 shows a typical force changing profile detected on two sandals, when a user walks four steps. At time $T_{1}$, the force reading on the right sandal (the gray line) dropped below this pressure threshold, corresponding to the right foot being in the air. At time $T_{2}$, the force reading on the right sandal exceeded this pressure threshold (corresponding to right foot heel-strike on the ground) while the force 


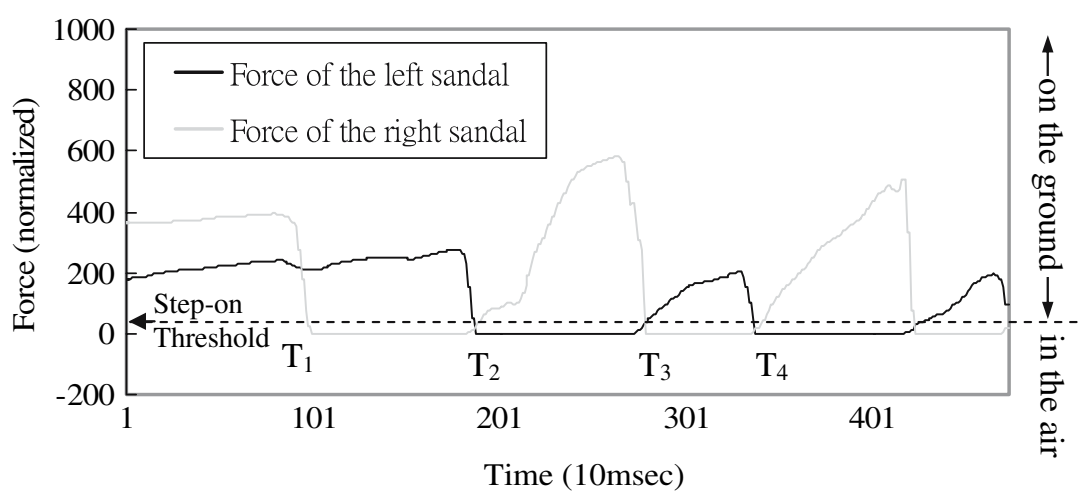

Fig. 8 Force changing profile collected on two force sensors installed underneath two sandals while a user walks four consecutive steps. At time $T_{1}$, a right foot is lifted off the ground. At time $T_{2}$, the right foot touches the ground in a heel-

reading of the left sandal (the black line) dropped below this pressure threshold (corresponding to left foot toe-off). We can see similar heel-strike and toe-off stances at $T_{3}$ and $T_{4}$.

We have undertaken two prototype implementations of the ultrasound-based distance measurement. In our first prototype [7], components for ultrasonicinfrared transmitters and receivers are obtained by disassembling the NAVInote's [27] electronic pen and base unit. NAVInote's positional accuracy is superb about a millimeter. Unfortunately, NAVInote's sensing range is limited to A4 size paper (about $30 \mathrm{~cm}$ long), which is too small for distance measurements since our method requires the sensing range to be larger than twice of the normal footstep size.

In our second prototype, Crossbow Cricket Motes [26] with a much longer measurement range (up to $10 \mathrm{~m}$ ) are used, but they have less positional accuracy. Two ultrasound-RF Cricket receivers are placed on the left sandal, and one ultrasound-RF Cricket transmitter is placed on the right sandal. These transmitter and receivers are positioned on the inner sides of the sandals and aligned to face each other to enable range estimation. In addition, we have found that ultrasonic transducers [28] on the Cricket unit are directional, meaning that distance measurement is accurate only when the angle difference between a transmitter unit and a receiver unit is within $90^{\circ}$. However, in a normal walking motion, this angle difference can go up to $180^{\circ}$ at heel-strike and toe-off stances, resulting in inaccurate distance measurements. Therefore, we have modified the Cricket transmitter unit by soldering three ultrasonic transducers together, facing three of the four directions and, enabling wider angle coverage of ultrasonic pulses. In addition, we have modified the distance measurement procedure in the Cricket firm- strike stance, and the left foot is lifted up in a toe-off stance. At time $T_{3}$, the left foot enters a heel-strike stance and the right foot enters a toe-off stance. The state at time $T_{4}$ is the same as the state in time $T_{2}$

ware to trigger measurements only at the heel-strike and toes-off stances (e.g., both feet touching the ground).

For the accelerometer-based method, a Phidgets dual-axis accelerometer is installed underneath a left sandal. The maximum sensing range is $\pm 2 \mathrm{~g}$ and the adopted sensitivity is $0.05 \mathrm{~m} / \mathrm{s}^{2}$. In addition, we have chosen $\pm 0.8 \mathrm{~m} / \mathrm{s}^{2}$ as a vibration noise filtering threshold based on several trial-and-error tests.

For both distance measurement methods, an orientation sensor is needed. The ultrasound based method uses the orientation angle to re-orient a vector from a local coordinate system to a global one, whereas the accelerometer based method uses the orientation angle to filter out the effect of gravitational acceleration. The orientation sensor used is the InterSense InterTrax2 [29], placed in the left sandal. The InterTrax2 is traditionally used as a head tracker in augmented reality applications, providing 3-DOF (degrees of freedom) angular tracking. From its product specification, it can achieve $0.02^{\circ}$ relative angular resolution.

For passive RFID grid calibration, a Skyetek M1 [30] RFID reader is installed at the bottom of the left sandal. In addition, a set of passive RFID tags with a read range of $4.5 \mathrm{~cm}$ are placed in a grid fashion in the environment.

\section{Experimental results}

To evaluate the positional accuracy of our GETA prototype, we designed three different experimental scenarios. Figure 9 shows the floor map of our experimental environment on the third floor of the CSIE building at National Taiwan University (NTU). The first scenario consists of five human test subjects, who 
Fig. 9 The figure shows the experimental traces in NTU CSIE building. The straight line experiment starts from point $X$ to point $Y$. The rectangular trace experiment starts from point $A$, following a counterclockwise rectangular loop direction through points $B, C, D, E$, and then backs to $A$

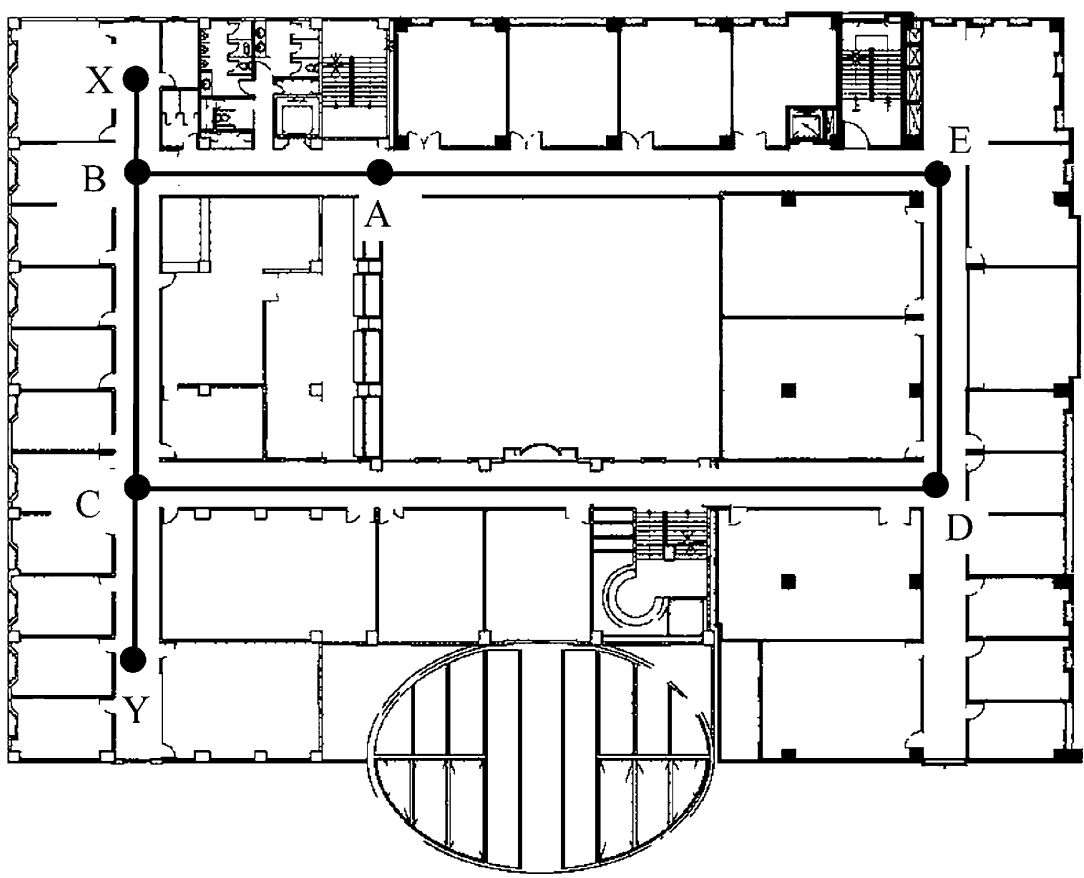

walked a $30-\mathrm{m}$ straight line from point $\mathrm{X}$ to point $\mathrm{Y}$. We then observe how well GETA sandals perform in positional accuracy along this path. The second scenario consists of the same five students who walked a $117 \mathrm{~m}$ rectangular loop. Starting from point A, the subjects, followed a counter-clockwise loop to points B, C, D, E, and finally backed to point A. The third scenario consists of the same five students climbing $10 \mathrm{~m}$ of stairs, starting from the 3rd floor to the 4th floor.

\subsection{Scenario I: straight line}

In the first scenario the five subjects for GETA sandals walked over a designated $30 \mathrm{~m}$ straight path three times, each time using a different walking style. These three walking styles are defined in Table 1 with different cadences and velocities. For example, in the first walking style, each subject walked with an approximately $0.3 \mathrm{~m}$ uniform stride length at an average speed of one step per second, which translated into a velocity of $0.3 \mathrm{~m} / \mathrm{s}$. To enable our subjects to conform easily to

Table 1 Three different walking styles settings for $30 \mathrm{~m}$ straight line experiment

\begin{tabular}{lllll}
\hline $\begin{array}{l}\text { Walking } \\
\text { Styles }\end{array}$ & $\begin{array}{l}\text { Cadences } \\
(\text { steps/s) }\end{array}$ & $\begin{array}{l}\text { Velocities } \\
(\mathrm{m} / \mathrm{s})\end{array}$ & $\begin{array}{l}\text { Stride } \\
\text { lengths }(\mathrm{m})\end{array}$ & $\begin{array}{l}\text { Number } \\
\text { of steps }\end{array}$ \\
\hline 1 & 1 & 0.30 & 0.30 & 100 \\
2 & 1.17 & 0.41 & 0.30 & 100 \\
3 & 2 & 0.60 & 0.30 & 100 \\
\hline
\end{tabular}

the designated uniform step size, visible markers were taped on the floor. Testing uniform stride length enables easy measurement of step-by-step positional errors. Note that the tested uniform stride length $(0.3 \mathrm{~m})$ is shorter than a normal size of a step (approximately $0.75 \mathrm{~m}$ ) of an average-height adult wearing comfortable shoes. This shorter stride length is due to the form factor of the current GETA sandals prototype. Also note that our system also works under non-uniform stride length.

Figure 10 plots average positional errors of these five test subjects using our footstep system and ultrasound-based distance measurement. Three plotted curves correspond to the three different walking styles in Table 1. All three curves show the problem of error accumulation. They also show that walking style \#1 performs best with an average positional error of $3.52 \mathrm{~m}$, walking style \#2 follows with an average positional error of $5.87 \mathrm{~m}$, and walking style \#3 is worst with an average positional error of $9.10 \mathrm{~m}$. Figure 10 shows a positive correlation between cadence and average positional error, i.e., a higher stepping rate (cadence) leads to a larger average positional error.

This correlation can be explained by how GETA sandals detect heel strike motion. In a heel strike motion, the heel strikes the ground first, followed by the toe. Since the force sensor is installed on the toe, GETA sandals won't detect the heel strike stance until the toe reaches the ground. Under a fast stepping rate, when the toe strikes the ground, the other foot may have already started moving forward. In other words, this delay in 
measurement may lead to under-estimation of step vectors. We have observed different amounts of delayed underestimation errors from different human test subjects, which depend on their habitual walking motions. This adverse underestimation effect can be addressed by a simple redesign of GETA sandals, i.e., by repositioning the force sensor to the heel.

Figure 11 plots the average positional errors of five subjects using our footstep system and accelerometerbased distance measurement. This case assumes that obstacles are blocking ultrasonic pulses between two sandals. Three plotted curves correspond to the three different walking styles in Table 1 . These curves show that walking style \#1 performs best with an average positional error of $6.41 \mathrm{~m}$, walking style \#2 follows with an average positional error of $7.19 \mathrm{~m}$, and walking style \#3 is worst with an average positional error of $9.21 \mathrm{~m}$. Similar to results in ultrasound-based distance mea- surement, Fig. 11 also shows a positive correlation between cadence and average positional error. One possible explanation for this correlation is that a higher stepping rate (a smaller time interval between two successive steps) leads to fewer acceleration samples per step vector measurement. Since a step vector is estimated by computing double-integral of these discrete accelerometer samples, a smaller number of samples leads to less accurate step vector estimation.

Under the straight line walking scenario, the ultrasound-based distance measurement achieves a better average positional accuracy $(20.5 \%$ average positional error) than the accelerometer-based distance measurement (25.3\% average positional error). This shows that the accelerometer-based method works relatively well, and it can provide a viable distance measurement in those cases where the ultrasound-based method fails.
Fig. 10 The average positional error of five human straight line, in three different walking styles and using ultrasound-based distance measurement subjects walking a $30 \mathrm{~m}$

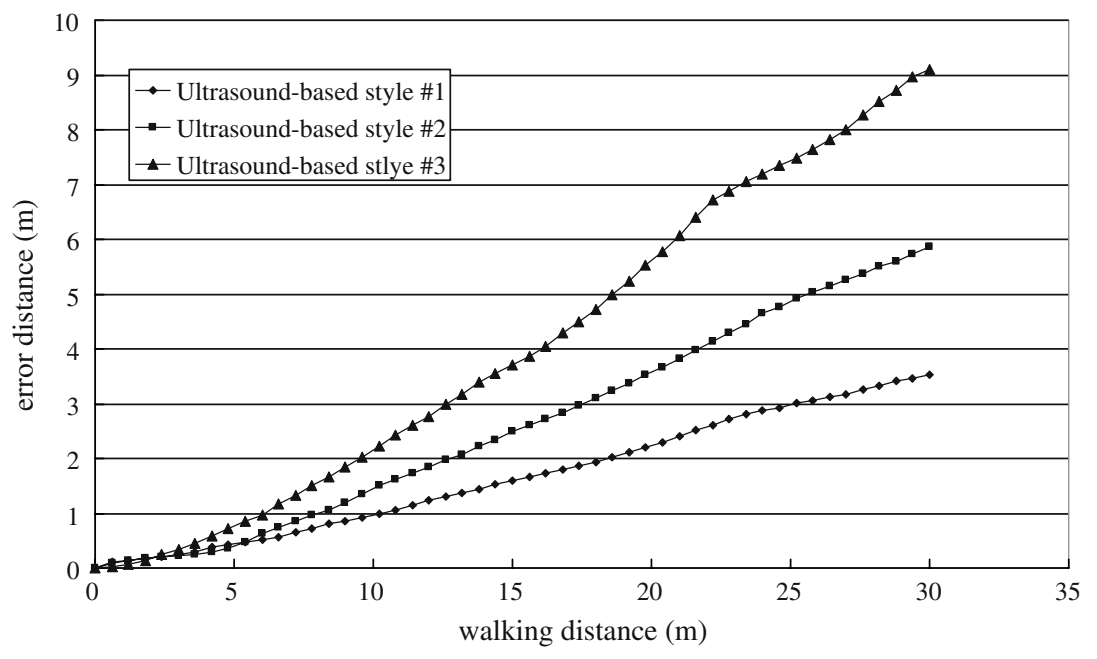

Fig. 11 The average positional error of five human subjects walking a $30 \mathrm{~m}$ straight line, in three different walking styles and using accelerometer-based distance measurement

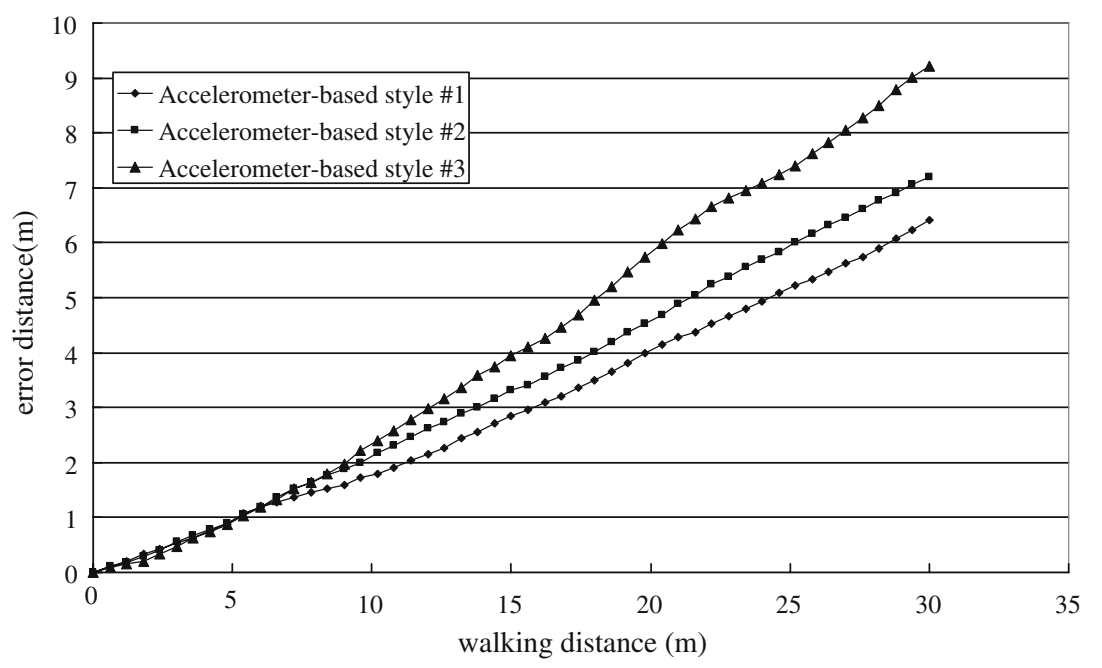




\subsection{Scenario II: rectangular loop}

In the second scenario the same students walk three times with GETA sandals over a designated $117 \mathrm{~m}$ rectangular loop, each time using a different walking style, defined in Table 2. To enable subjects to conform easily to the designated uniform step size, visible markers are taped on the floors.

Figure 12 plots the average positional errors of subjects using our footstep system and ultrasound-based distance measurement. Three plotted curves correspond to the three different walking styles in Table 2 . These curves show that walking style \#1 performs best with an average positional error of $7.54 \mathrm{~m}$, walking style \#2 follows with an average positional error of $10.00 \mathrm{~m}$, and walking style \#3 is worst with an average positional error of $13.75 \mathrm{~m}$. These results support the observed trends from the previous scenario that is a positive correlation between cadence and average positional error. Note that some segments of curves, specifically point $\mathrm{E}$ to point $\mathrm{A}$, show minor exception to this trend. The reason for this exception is that some rooms along the corridor from point $\mathrm{E}$ to point $\mathrm{A}$ emitted strong magnetic fields. This affects the accuracy of the orientation sensor.

Figure 12 shows that the average positional error and the rate of error growth decrease in two segments of the curves (C-D and D-E). This is due to the effect of error cancellation as subjects move past corners $\mathrm{C}$

Table 2 Three different walking styles for the $100 \mathrm{~m}$ rectangular loop experiment

\begin{tabular}{lllll}
\hline $\begin{array}{l}\text { Walking } \\
\text { Styles }\end{array}$ & $\begin{array}{l}\text { Cadences } \\
(\text { steps/s) }\end{array}$ & $\begin{array}{l}\text { Velocities } \\
(\mathrm{m} / \mathrm{s})\end{array}$ & $\begin{array}{l}\text { Stride } \\
\text { lengths }(\mathrm{m})\end{array}$ & $\begin{array}{l}\text { Number } \\
\text { of steps }\end{array}$ \\
\hline 1 & 1 & 0.30 & 0.30 & 398 \\
2 & 1.33 & 0.40 & 0.30 & 398 \\
3 & 2 & 0.60 & 0.30 & 398 \\
\hline
\end{tabular}

and D. In a rectangular loop, error cancellation occurs when subjects move back toward the direction of their starting points. This effect can be explained as follows: the accumulated positional error, which is generated from moving in the direction away from the starting point $\mathrm{A}$, is being canceled out by incoming positional error in the opposite direction, which is generated from moving toward the starting point $\mathrm{A}$.

\subsection{Scenario III: stair climbing}

The third scenario tests our footstep system under accelerometer-based distance measurement and during stair climbing. The same five students twice climb $10 \mathrm{~m}$ of stairs (18 steps) with GETA sandals, each time under the same velocity $(0.23 \mathrm{~m} / \mathrm{s})$, cadence $(0.91 \mathrm{step} / \mathrm{s})$, and uniform stride length $(0.35 \mathrm{~m})$.

Figure 13 plots the average positional errors of subjects using our footstep system and the accelerometerbased distance measurement. The curve shows an average positional error of $2.4 \mathrm{~m}$ over $10 \mathrm{~m}$ of stairs. We perform the same experiment using the ultrasoundbased distance measurement and observe a $71.42 \%$ failure rate, since stairs form sound-proof obstacles between two sandals. Therefore, for stairs our system must rely on accelerometer-based distance measurements.

\section{Conclusion and future work}

This paper describes the design, implementation, and evaluation of our footstep indoor location systems. Our footstep system can significantly reduce the amount of infrastructure needed when deployed in indoor environments. Most simply, our location system is contained within mobile traditional Japanese GETA sandals, making it easy for deployment everywhere.
Fig. 12 The average positional error of five human subjects walking a rectangular loop in three different walking styles and using ultrasound-based distance measurement. Points $A, B, C$, $D$, and $E$ are defined in Fig. 9

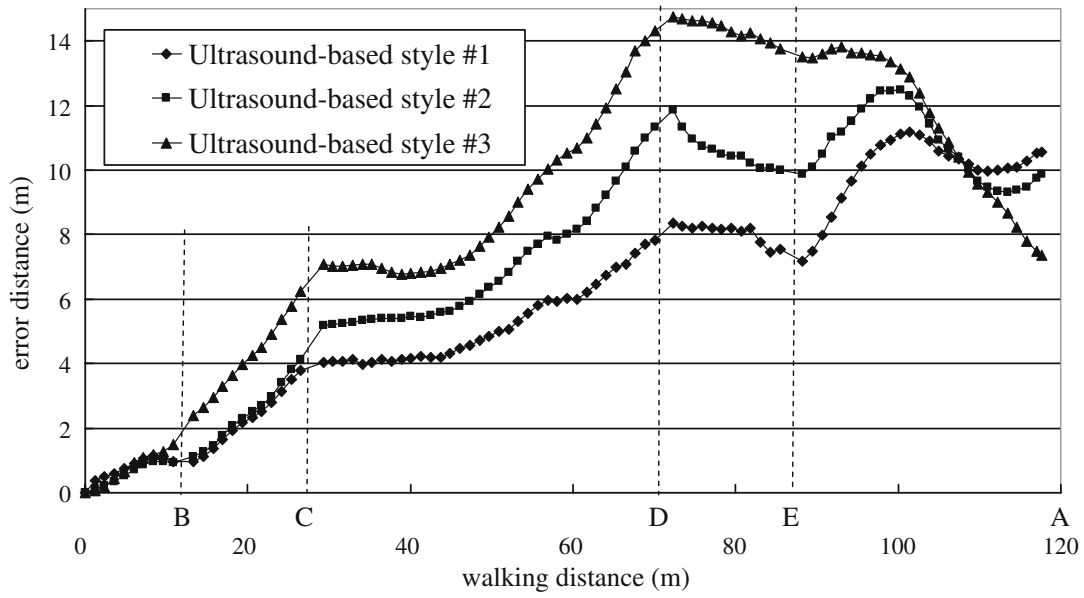




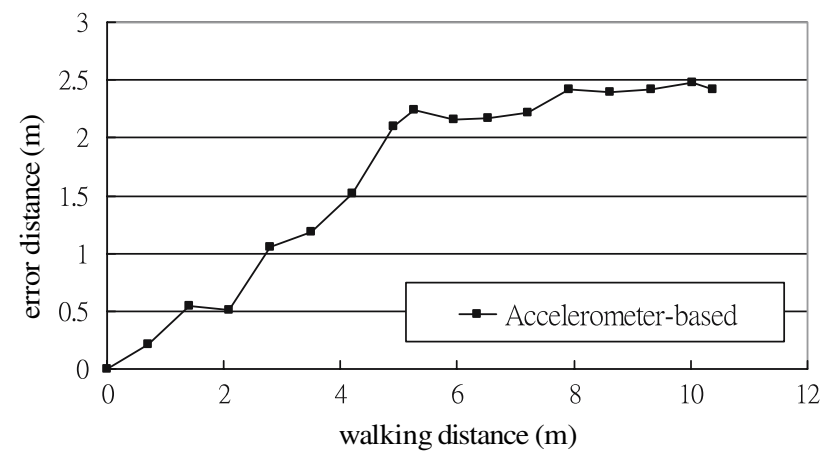

Fig. 13 The average positional error of five human subjects climbing $10 \mathrm{~m}$ of stairs and using the accelerometer-based distance measurement

The user simply wears GETA sandals to enable tracking of his/her location relative to a starting point. In addition to the benefit of low infrastructure cost, the footstep based method has fewer problems than other infrastructure-based indoor location systems such as noises, obstacles, interferences, and dead spots. Although the footstep based method achieves certain amount of accuracy per moving footstep, we have encountered several problems. (1) Since the positional error is accumulated over the distance traveled, it may need to be combined with a light RFID infrastructure to correct its positional error over longer distances. (2) Since the footstep-based method has limitations in situations such as stair climbing and jumping motions, our system incorporates accelerometer-based distance measurement to improve the robustness.

There are two additional problems in our current prototype of GETA sandals: wear-ability and RFID tag placement. The current wear-ability is unsatisfactory because all sensor components must be interconnected to a Notebook PC through hardwiring. In our next prototype, we would like to replace all hardwiring with wireless networking (e.g., Bluetooth), and replace processing on the notebook with a small embedded processor. To further reduce the RFID infrastructure, we are interested to locate RFID tags strategically in frequently visited spots in an environment. In addition, sensor fusion is also a target in our future work. The error distributions gathered from many tests would be very helpful. With the sensor fusion technique, we can further fuse the ultrasound and accelerometer based distance measurement to improve the accuracy of our footstep method.

\section{References}

1. Want R, Hopper A, Falcao V, Gibbons J (1992) The active badge location system. ACM Trans Inf Syst 10(1):91-102
2. Harter A, Hopper A, Steggles P, Ward A, Webster P (1999) The anatomy of a context-aware application. In: Proceedings of 5th MOBICOM, pp 59-68

3. Priyantha NB, Chakraborty A, Balakrishnan H (2000) The cricket location-support system. In: Proceedings of the 6th MOBICOM, Boston, MA, USA,

4. Orr RJ, Abowd GD (2000) The smart floor: a mechanism for natural user identification and tracking. GVU Technical Report GIT-GVU-00-02

5. Bahl P, Padmanabhan V (2000) RADAR: an in-building rfbased user location and tracking system. In: Proceedings of the IEEE INFOCOM, pp 775-784

6. Ekahau, Inc., "Ekahau Technology and Products", http:// www.vtt.fi/virtual/navi/expo2003/Ekahau030402.pdf

7. Okuda K, Yeh S-y, Wu C-i, Chang K-h, Chu H-h (2000) The ETA sandals: a footprint location tracking system, workshop on location- and context-awareness (LoCa 2005), in Cooperation with Pervasive 2005, (also published as Lecture Notes in Computer Science 3479, Location- and ContextAwareness), Munich, Germany, pp 120-131

8. UbiSense, http://www.ubisense.net

9. ITRI, http://www.itri.org.tw

10. Seshadri V, Zaruba GV, Huber M (2005) A bayesian sampling approach to in-door local-ization of wireless devices using received signal strength indication. In: IEEE conference on pervasive computing and communications (PerCom)

11. Hightower J, Borriello G (2005) Particle filters for location estimation in ubiquitous computing: a case study. international conference on ubiquitous computing

12. Schulz D, Fox D, Hightower J (2003) People tracking with anonymous and id-sensors using rao-blackwellised particle filters. In: International joint conference on artificial intelligence, pp 921-926

13. Fox D, Hightower J, Liao L, Schulz D, Borriello G (2003) Bayesian filtering for location estimation. IEEE Pervasive Comput 2(3):24-33

14. Paramvir Bahl A, Balachandran VN (2000) Padmanabhan: enhancements to the RADAR user location and tracking system. Technical report of Microsoft Research

15. ChenY-C, Chiang J-R, Chu H-h, Huang P, Tsui AW (2005) Sensor-assisted Wi-Fi indoor location system for adapting to environmental dynamics. In: ACM/IEEE international symposium on modeling, analysis and simulation of wireless and mobile systems (MSWIM 2005). Montreal, Quebec

16. Nebot EM (1999) Sensors used for autonomous navigation. In: Tzafestas SG (ed) Advances in intelligent autonomous systems. Kluwer, Dordrecht, pp 135-156

17. Roston GP, Krotkov E (1991) Dead reckoning navigation for walking robots. Technical Report CMU-RITR- 91-27, Robotics Institute, Carnegie Mellon University, Pittsburgh, PA

18. Foxlin E, Durlach N (1994) An inertial head-orientation tracker with automatic drift compensation for use with hmd's. In: Singh G, Feiner SK, Thalmann D (eds) Virtual reality software and technology: proceedings of the VRST'94 conference, World Scientific, London, pp 159-173

19. Welch G, Foxlin E (2002) Motion tracking: No silver bullet, but a respectable arsenal. IEEE Comput Graph Appl 22(6):24-38

20. Lee SW, Mase K (2001) Incremental motion-based location recognition. In: Proceedings of 5th international symposium on wearable computers. IEEE CS Press, Los Alamitos, pp $123-130$

21. GPS Conference (1997) Personal dead reckoning module. In the Institute of Navigation's GPS Conference, September 1997 
22. S Itiro, http://www.siio.jp/projects/idcarpet/index.html

23. Willis S, Helal S (2006) A passive RFID information grid for location and proximity sensing for the blind user, University of Florida Technical Report number TR04-009

24. Amemiya T, Yamashita J, Hirota K, Hirose M (2004) Virtual leading blocks for the deaf-blind: a real-time wayfinder by verbal-nonverbal hybrid interface and high-density RFID tag space. In: Proceedings of the 2004 virtual reality (VR'04)

25. Greenberg S, Fitchett C (2001) Phidgets easy development of physical interfaces through physical widgets. In: Proceedings of the UIST 2001 14th annual ACM symposium on user interface software and technology, ACM Press, Orlando, Florida, 11-14 November 2001, pp 209-218

26. Baliga RB (2004) Rapid coordinate system creation and mapping using crickets. M. Eng. Thesis, Massachusetts Institute of Technology

27. NaviNote technology, http://www.navinote.com

28. Kobitone Ultrasonic Transducer, http://www.mouser.com/ index.cfm?handler=displayproduct\&lstdispproductid $=302497 \&$ e_categoryid $=402 \&$ e_pcodeid $=02505$

29. InterSense, http://www.isense.com

30. Skyetek RFID engineering, http://www.skyetek.com/ index.php 